\title{
Active and Smart Ageing: The Use of Technology for Preventive Medicine
}

\author{
Constantina Alvarez* \\ Electrical Engineering Department, Gijon, Mechanical Engineering \& Mechatronics - Hochschule Karlsruhe, Spain \\ *Corresponding author: Constantina Alvarez, Electrical Engineering Department, Gijon, Mechanical Engineering \& Mechatronics \\ - Hochschule Karlsruhe, Spain
}

\begin{tabular}{|c|c|}
\hline ARTICLE INFO & ABSTRACT \\
\hline Received: 幽 June 07, 2019 & The present review shows the need of advances in data analysis of stored informa- \\
\hline Published: 蔧 June 13, 2019 & $\begin{array}{l}\text { tion gathered from existing wearable health tracking technologies and the need of AI } \\
\text { data interpretation based on large collections of data for positive and accurate predic- }\end{array}$ \\
\hline
\end{tabular}

Citation: Constantina Alvarez. Active and Smart Ageing: The Use of Technology for Preventive Medicine. Biomed J Sci \& Tech Res 18(5)-2019. BJSTR. MS.ID.003209.

\section{Introduction}

Public health diseases affect people across the world in early and older ages. The early detection of any anomalies in the life of a person is compulsory in the process of saving patient's lives. Aging and unhealthy lifestyles are just some of the factors that worsen health in humans. These factors deteriorate life quality increasing the risk of development dangerous diseases. Among the leading causes of dead in the world one can find Tuberculosis, Alzheimer's disease, Diabetes, Cancer, Chronic Obstructive Pulmonary Disease (COPD), Stroke, Ischemic heart disease (coronary artery disease) etc. Some of these diseases do not have noticeable symptoms in their early stages of development and only preventive medicine has shown to be the way to reduce the chances of getting ill [1].

As it is known, medicine has long been applied to cure and not until few decades ago to prevent. Yet, together with the development of medicine and the uprising discoveries of science and technological advances of this time, medicine alone has help prolong human life expectancy in a significant way [2]. It is the case of detecting heart anomalies in Cardiology, Cancer in Oncology or Diabetes in Endocrinology. Prevention is key to avoid health problems and that is where doctors have played an important roll recommending their patients in a daily basis to stay healthy, to eating property, to exercise, to get tested frequently, and to do as much as possible to find any anomalies in their bodies. This is just the start point for preventive medicine. With the introduction of new and affordable technologies that efficiently and without side effects can diagnose anomalies, medicine is starting a new era of active and smart aging.

Initiatives like the AAL program (Active Assisted living programme) founded by the European Union are examples of modern aging ways. This programme intend to fund innovation that keeps people healthy happy and helps everyone get on with old age [3]. The idea goes around developing products and services that make a real difference in people's lives. Some examples of these applications are rehabilitation gaming systems for post-stroke patients or robot assistants for improving wellbeing of older adults. This is substantial and more is to come. Affordable technologies have allowed us to have access wearable technologies, smart 'on' mode tracking vital signs devices that store big amounts of data. Wearable computers that are in fact connected devices always 'on' mode to make the appropriate measurements in real time [4].

Much is unknown of how using commercial wearable "health" tracking devices that store vital data signs, can be used to detected early and effectively the propensity to develop any health issues since they remain technologically limited. This limitation can be overcome in the near future addressing limitations like battery life, chipset limitation and design matters [5]. Vital health data analysis will rely on big data to advance medical science to create better diagnostics, drugs and develop active preventive medicine. 


\section{Getting the Data}

One example in cardiology is the use of precordial signals using 3D seismocardiography (SCG-3D) for the detection of anomalies. Seismocardiography (SCG) is the measurement of chest vibrations (precordial area) produced by contraction and ejection of blood from the ventricles to the vascular tree [6]. For the measurement it is necessary to establish the exact location in the precordial area, to place the sensor correctly (3-axis accelerometer), identify the SCG signal using digital filters. The analysis of the dorso-ventral axis and the acquisition system, where the data is stored for further processing using software, machine learning algorithms can assess clinical status of heart failure patients [6]. The sismocardiography as a biomedical device to measure cardiac activity is a great option in the detection of cardiac problems prematurely, as well as in the help of cardiac resynchronization therapies and other types of diseases that are mentioned in many studies, such as coronary problems, chronic heart problems, among others.

SCG measurements is an example of the advances in technology. This SCG measurements and results can be compared with analyses such as the Echocardiogram to evaluate the progress of patients who undergo cardiac resynchronization treatments or heart failure. The difference between having a 'on' mode SCG analysis and an Echocardiogram is the cost and the frequency of the measurement. Due to the expensive equipment and the difficulty of performing frequent clinical examinations like the Echocardiogram, it is not very viable, so the SCG with the possibility of performing simple, constant and effective examinations makes it a great option for a wearable heath device.

3-axis accelerometer sensors are cheap and are being use today by activity tracking devices commercially marketed and use today. Most of these devices do not track the heart rate with the accelerometer sensors. Accelerometers track activity, steps and exercise using the information collected interpreting it with algorithms that include user's information GPS data and other inputs to calculate distance, speed, burned calories etc. Hence, data analysis. To track heart rate, most trackers work with pulse oximetry technology. This technology uses an infrared light that penetrates the skin and reviews changes in the color of the blood. These colors represent the oxygenation of the blood. From there that when blood is more oxygenated heartbeat is higher. This heart rate info is also use to calculate the output information. And this is where it gets interesting. Even though these wearable devices are already been use, these technologies are far from accurate and even farther to be call health devices to collect accurate data.

Interesting examples of wearable today used health devices are the artificial units that monitors blood glucose levels automatically and injects insulin to a defined level [7]. However, these glucose trackers are only used by those who already have a condition. To start getting a benefit for the development of preventive medicine this type of technology needs to be affordable as with the activity and heart rate trackers. Healthcare research has always depended on collection of accurate data and analysis. Wearable technologies will help provide a solid platform to share patient's data to take necessary actions to process that data and remotely in real time keep an eye on health with the help of property trained doctors or algorithms.

Preventive medicine will depend on remote measurement of patient status made with wearable sensors. Live updates regarding patient status will be available for doctors and users all the time. Patients will have the facilitates to get feedback anywhere without having to even visit the doctor. This will empower patients to selfmanage health therefore preventing in most cases. But before all of this we have to start getting the data. The accurate data.

\section{Smart Aging Systems}

The monitoring of vital signs and lifestyles during life time is the way to go to implement preventive medicine in our life. Facilitate doctors to remotely categorize conditions or give an insight into symptoms of any disease will provide health improvement in advice and prevent health disease conditions in advance [8]. The in-depth study of the physical and living environment characteristics of the human body interaction, especially those proved to be the leading causes of mortality risk, will make possible the relation between data collected and actual diseases to accurately monitor and control outcomes through technological systems, implementing the latest advances in electronic design techniques, signal processing and software engineering. The advance of biomedical instrumentation to measure cardiac activity, blood composition and pressure, together with environment heterogeneous sensory data will help to improve the anomaly health detection over the life time of a person. Making life time data history of health vital signs indispensable for well-being monitoring and anomaly detection. True preventive medicine. Opportunities for improvements in algorithms for detecting are the starting point and completion of the analysis of each condition must be acknowledged in the process of getting data to create accurate and functional smart aging systems that offer results.

\section{Conclusion}

It is observed that it is feasible to develop an integrated system for the acquisition of individual and external data, which are then sent to a computer for processing and later visualization. It is possible to determine that the result is valid, but it is necessary to improve the collection system and technology, as the processing algorithm, since there were some differences and gaps in the research area. It was possible to provisionally identify in this first phase, many of the important points of the future outcome wave, as well as starting the recording database of patients, to enter the validation phase of and smart aging system that will promote active and healthy lifestyles.

\section{References}

1. Mathers, Colin D (2005) Counting the dead and what they died from: an assessment of the global status of cause of death data. Bulletin of the world health organization 83(3): 171-177. 
2. Lee, Ronald (2019) Mortality forecasts and linear life expectancy trends Old and New Perspectives on Mortality Forecasting. Springer Cham pp. 167-183.

3. (2007) AAL: The Ambient Assisted Living Joint Programme.

4. Morabito, Vincenzo (2016) Wearable technologies: The future of digita business innovation. Springer, Cham, 23-42.

5. Howard (2015) Wearable technology trends in 2015 - Apadmi. Apadmi

ISSN: 2574-1241

DOI: 10.26717/BJSTR.2019.18.003209

Constantina Alvarez. Biomed J Sci \& Tech Res

(c) (P) This work is licensed under Creative Commons Attribution 4.0 License

Submission Link: https://biomedres.us/submit-manuscript.php
6. Inan OT, Baran Pouyan M, Javaid AQ Dowling S, Etemadi M, et al. (2018) Novel wearable seismocardiography and machine learning algorithms can assess clinical status of heart failure patients. Circulation: Heart Failure 11(1): 004313.

7. Bruen Danielle, Delaney C, Florea L, Diamond D (2017) Glucose sensing for diabetes monitoring: recent developments. Sensors 17(8): 1866.

8. Ghayvat H, Awais M, Pandya S, Ren H, Akbarzadeh S, et al. (2019) Smart aging system: Uncovering the hidden wellness parameter for well-being monitoring and anomaly detection. Sensors 19(4): 766.

$\begin{array}{ll}\text { BIOMEDICAL } & \text { Assets of Publishing with us } \\ \text { RESEARCHES } & \text { - Global archiving of articles } \\ & \text { - Immediate, unrestricted online access } \\ \end{array}$

